\title{
Spontaneous reactions to health risk feedback: a network perspective
}

\author{
Martina Panzer • Britta Renner
}

\begin{abstract}
Research on the reception of health risk feedback has focused on the analysis of single, researcher-selected cognitive reactions. The full range of spontaneous reactions and their patterns have received little attention. The present paper explores content, interrelations, and adaptivity of spontaneous reactions to health risk feedback from a network perspective. Participants $(n=423)$ received blood pressure and cholesterol feedback and listed their thoughts afterwards. A network of reactions to health risk feedback was constructed from the responses. Emotions, risk feedback valence, future lifestyle, and expectedness emerged as strong and largely well-connected network nodes, while previously well-researched reactions like feedback acceptance formed small, less connected nodes. The majority of reaction patterns identified through the network appeared adaptive, even after negative feedback. The network provides a potentially useful tool for research and practice, highlighting previously neglected relevant reactions, and providing a group-level background against which individual reactions can be evaluated.
\end{abstract}

Keywords Health communication - Risk perception · Risk information processing - Reactions to risk information

\footnotetext{
M. Panzer

Jacobs Center for Lifelong Learning, Jacobs University Bremen, Campus Ring 1, 28759 Bremen, Germany

e mail: m.panzer@jacobs university.de

B. Renner ( $\square)$

Department of Psychology, University of Konstanz,

P.O. Box 47, 78457 Konstanz, Germany

e mail: britta.renner@uni konstanz.de
}

\section{Introduction}

Cardiovascular disease (CVD) is the major cause of death worldwide (WHO 2004) with elevated blood pressure and cholesterol levels being main risk factors for CVD in Western and in Asia-Pacific countries (WHO 2002; Asia Pacific Cohort Studies Collaboration [APCSC] 2002, 2005). As a consequence, national health organizations as well as commercial companies are steadily extending their health risk screening programs. However, most national health organizations focus on effectiveness, monitoring, and management of screenings whereas the question how people react toward receiving threatening risk information appears ever more urgent. Since the range and number of health screening tests is rapidly increasing (e.g., through the rise of genetic testing, cf. Pear 2008) it appears utterly important to better understand lay people's behavioral and psychological reactions toward health risk information.

Reactions towards health risk screenings

Most field studies and laboratory experiments addressing reactions to individual health risk feedback have studied predominantly cognitive reactions, particularly perceived test result accuracy and the degree of message acceptance (e.g., Ditto and Lopez 1992; Ditto et al. 1998). Commonly, these cognitive reactions have been assessed by asking respondents a pre-defined set of closed questions (e.g., "How confident are you that this test result is an accurate indication of your actual [...] status?", Ditto et al. 1998, p. 63). Supporting the notion of self-defensively biased reactions, various studies showed that people receiving bad news rated their test result as less accurate than people receiving good news (Ditto et al. 2003; Taylor 1991). A recent study examining reactions of individuals who took 
part in a cholesterol screening replicated this finding of selfdefensive reactions toward risk feedback (Croyle et al. 2006). Participants were asked to recall their cholesterol measure 6 months after the screening. Results showed that participants who received the most undesirable test results were most likely to remember their cholesterol scores as lower (i.e., healthier) than the scores they actually received. These findings were interpreted by the authors as evidence that reactions toward self-relevant health information are susceptible to self-defensive biases.

While these studies greatly facilitated the understanding of risk feedback reception, there are also limitations when asking a pre-defined set of questions (Sudman et al. 1996; Weiner 1985). Most importantly, the method is reactive and participants may answer questions regarding aspects of risk feedback which are not part of their spontaneous reaction repertoire. Furthermore, it is also possible that important aspects of feedback reception which might be central for coping and behavioral processes are not assessed. Supporting this notion, a recent study assessing spontaneous responses to cholesterol and blood pressure risk feedback showed that cognitive reactions such as acceptance of feedback, which were typically assessed in previous research, represent only a small portion of the total array of spontaneous reactions (Panzer and Renner 2008). More specifically, if reactions are not pre-selected by the researcher, but generated freely by the recipients, the predominant type of reaction appears to be emotions, followed by reactions referring to the valence or expectedness of the given feedback, or to lifestyle changes. These findings indicate which types of spontaneous reactions prevail after receiving risk feedback. However, they are silent in respect to how these different types of reactions are related.

\section{Interrelations between reactions toward risk feedback}

In previous research the focus has been on specific, single reactions. However, focusing on the interrelations between reactions might yield a dramatically different picture. In an experimental study on reactions toward (fictitious) cholesterol test results, respondents who had received an elevated cholesterol test result rated the test as less accurate and cholesterol as less serious than respondents who had received a normal cholesterol test result. Croyle et al. (1993) interpreted this pattern of results as clear evidence for selfdefensive reactions toward unfavorable information. However, the same recipients of an unfavorable test result were also more inclined to change their behavior. Accordingly, participants receiving bad news appeared to be more skeptical toward the quality of the information but were simultaneously more motivated to change their behavior, indicating an adaptive reaction pattern rather than a selfdefensive one driven by a desire to derogate unpleasant information. A similar pattern of results has been observed in a public cholesterol screening (Renner 2004). Specifically, participants receiving a test result indicating an elevated cholesterol level were more skeptical concerning the reliability of the test result than those with an optimal cholesterol test result. Nevertheless, $42 \%$ of the former reported that they had changed their behavior at a 6 month follow-up after the cholesterol screening. These results suggest that a joint analysis of different types of reactions is required for distinguishing maladaptive, self-defensive from adaptive reactions toward risk feedback.

A new approach for analyzing reactions toward risk information: a network perspective

In order to analyze the different aspects of reactions toward risk information conjointly, such as the observed types of reactions and the relationships between them, we suggest a new approach, i.e., a network perspective. We use the term network to refer to a simultaneous analysis of a set of variables (namely reaction types) and their interrelations which are then graphically represented as nodes and paths (cf. Colman 2006; Heckerman 2001). The network visualizes four different types of information about the observed reactions toward risk information. Firstly, the network includes information about the various dimensions or types of reactions generated by the respondents (e.g., emotions, cognitions about lifestyle changes) represented by different nodes. Secondly, for tailoring practical interventions such as doctor patient counseling, it is important to assure that prevalent reactions receive a greater weight in analysis and intervention than reactions which occur comparably seldom. Accordingly, the prevalence indicating the practical significance of each type of reaction is represented in the network by the proportional size of the respective network node. Thirdly, the specific content of the observed reaction type is represented in the network as proportional segment of the respective network node. For example, the emotion dimension might entail positive and negative emotions, thus its node might have two different segments. The more frequently generated one would be represented by a proportionally larger segment. Finally, the co-occurrences between different reaction types are represented through connections between network nodes, reflecting their frequency relative to other interrelations between the nodes.

\section{The present study}

The overarching aim of the present study was threefold: (1) to gain information about spontaneous reactions to individual health risk feedback in a real-life setting, (2) to 
explore their pattern of interrelations by developing a network of reactions to health risk information including the type, prevalence, and segmentation of reactions as well as the co-occurrence of different reaction types, and (3) to explore the network of reactions in order to identify reaction patterns which appear more or less adaptive in relation to the risk feedback received. Furthermore, reactions toward two different types of risk feedback were assessed, i.e., blood pressure and total cholesterol level feedback. Thus, reliability of reaction patterns across different types of risk feedback was additionally examined.

\section{Method}

Procedure

The present study is part of the larger research project risk appraisal consequences in Korea (RACK), during which two community-based blood pressure and cholesterol screenings were conducted with a time lag of 6 months in between. Participants were recruited via information leaflets and flyers in multiple institutions (e.g., universities, parishes) located in Seoul, South Korea. At both screenings, trained laboratory assistants measured participants' blood pressure and total cholesterol levels. The assistants provided each participant with a standardized oral feedback in Korean, along with an individual feedback sheet which contained the exact measured values in numbers (cholesterol level in $\mathrm{mg} / \mathrm{dl}$, systolic and diastolic blood pressure in $\mathrm{mmHg}$ ) and an additional graphical classification of each value as optimal, borderline high, or high in accordance with international standards (WHO 2004). ${ }^{1}$ Blood pressure readings of $\leq 140 / 90 \mathrm{mmHg}$ and cholesterol readings of $\leq 200 \mathrm{mg} / \mathrm{dl}$ were classified as normal and described as posing no elevated risk for CVD. Systolic blood pressure readings of $141160 \mathrm{mmHg}$, diastolic blood pressure readings of $9195 \mathrm{mmHg}$, and total cholesterol levels of $201240 \mathrm{mg} / \mathrm{dl}$ were classified as borderline high. Systolic blood pressure $>160 \mathrm{mmHg}$ or diastolic blood pressure $>95 \mathrm{mmHg}$, and cholesterol levels of $>240 \mathrm{mg} / \mathrm{dl}$ were classified as high. Recipients of borderline high or high readings were informed about the potential risks of these readings for CVD. Directly after receiving cholesterol and blood pressure feedback, participants were asked to list any thoughts or ideas they had immediately after receiving their test results (Cacioppo and Petty 1981). This task was given separately for thoughts regarding the cholesterol and the blood pressure test result.

\footnotetext{
${ }^{1}$ The cut off for high blood pressure was chosen in consultation with faculty from the College of Medicine at Hanyang University in Korea.
}

In the present study, only data from the second screening are analyzed and reported. Details and results for the first screening are described by Panzer and Renner (2008) and by Renner et al. (2007).

\section{Participants}

Five hundred and ninety-seven volunteers took part in the second screening, out of 951 who took part in the first screening half a year earlier (cf. Panzer and Renner 2008). Only participants reporting at least one spontaneous reaction were included in the main analyses. The final sample thus consists of 423 responders ( $51 \%$ women) with a mean age of 31 years $(\mathrm{SD}=18)$. Responders had an average cholesterol level of $160 \mathrm{mg} / \mathrm{dl}(\mathrm{SD}=22)$, which is below the mean South Korean population cholesterol level of $187 \mathrm{mg} / \mathrm{dl}$ (cf. Kang et al. 2006). Among the 8\% of responders with non-optimal cholesterol levels, the mean level was $227 \mathrm{mg} / \mathrm{dl}(\mathrm{SD}=22 \mathrm{mg} / \mathrm{dl})$. The responders' mean blood pressure level was $122 / 81 \mathrm{mmHg}$ which is comparable to the mean population level of $120 / 78 \mathrm{mmHg}$ (cf. Kang et al. 2006). Among the $14 \%$ of responders with non-optimal blood pressure, the mean was $148 / 94 \mathrm{mmHg}$ $(\mathrm{SD}=15 / 15 \mathrm{mmHg})$.

One hundred and seventy-four participants took part in the second screening but did not report any spontaneous reaction. These non-responders were on average 34 years old $(\mathrm{SD}=18)$ with $62 \%$ women; they had an average cholesterol level of $166 \mathrm{mg} / \mathrm{dl}(\mathrm{SD}=26)$ and an average blood pressure of 125/81 mmHg. Analyses comparing responders with non-responders showed that responders had a lower mean cholesterol level, $t(281.50)=2.67, p<.01, d=0.3$ and were more likely to be male, $\mathrm{X}^{2}(1)=6, p<.05$. However, the two groups did not differ significantly with regard to blood pressure or age $(t s(595)>1.9, \mathrm{~ns})$.

Differences in educational attainment may provide one explanation for non-response. Non-responders had a lower formal education (17\% no completion of high school, $46 \%$ high school degree, $37 \%$ college or university degree) than responders (11\% no completion of high school, $40 \%$ high school degree, $49 \%$ college or university degree), $X^{2}(2)=$ $6.44, p<.05$. The pattern found for responders is, however, more similar to South Korean high school enrollment and advancement rates of $97 \%$ and $85 \%$, respectively (Organization for Economic Co-Operation and Development 2005), than the pattern found for non-responders.

\section{Coding system}

Responses were translated from Korean to English by a bilingual Korean native speaker who was unaware of the purpose of the study. The coding system used in the present 
study is an extension of the risk feedback reaction coding system (RFR-system) developed by Panzer and Renner (2008) for coding responses to the first wave of the RACK screening. One coding unit comprised all words one participant wrote (e.g., "I was surprised with my high blood pressure reading") in response to the respective thought listing task (cholesterol, blood pressure). In a first step, each coding unit was examined as to whether it contained at least one reference to the screening result or topic. Each relevant unit (387 for cholesterol, 369 for blood pressure feedback) was rated on each dimension of the RFR-system, with each dimension representing one reaction type: (1) emotions (e.g., "I feel depressed"; "I am very happy"), (2) risk feedback valence (e.g., "I have a bad result"; "I received a normal reading"), (3) expectedness (e.g., "The result was unexpectedly high"; "I thought that I might end up with a high cholesterol level, but I am assured that it is not"), (4) future lifestyle (e.g., "I'll exercise more"; "I need to control my diet"), (5) causal attribution (e.g., "I lead a healthy life, so this could be expected"; "The results reflect my obsession with dried squids"), (6) implications for health (e.g., "It is an indication of poor health"; "I am in good health"), (7) need for information (e.g., "I want more precise results"; "I didn't understand what they were saying"), and (8) acceptance ("I will have a reexamination"). The present RFR-system was extended to include the additional dimension (9) comparison with previous results (e.g., "It's higher than in the past"; "It's the same as before"). These nine dimensions captured $91 \%$ of the total of relevant reactions generated. Additionally, a (10) residual category was available for coding units without reference to any of the nine dimensions, including $9 \%$ of the total of relevant reactions generated (e.g., "It was a good idea to be examined").

Extending previous results (Panzer and Renner 2008), mutually exclusive subcategories were coded for each of the nine reaction dimensions representing the segments of each network node. These subcategories had been developed on the basis of models of reactions toward individual health risk feedback (e.g., Leventhal et al. 2003; Ditto et al. 1988), and on reactions included in previous studies (e.g., Ditto et al. 2003; Croyle et al. 1993; Renner 2004; Renner 2003) supplemented by a review of $10 \%$ of thoughts listed (these responses were excluded from further analysis). For example, the dimension emotions entailed the subcategories (a) positive emotions (e.g., "I am happy"), (b) negative emotions (e.g., "I feel depressed"), (c) mixed emotions (e.g., "I feel mostly good, but still a little worried"), and (d) unclear emotions (e.g., "I'm not bothered too much").

Interrater agreement was substantial or almost perfect for all nine dimensions, with Fleiss' kappa from .62 to 1, and with percentage agreements ranging from $90 \%$ to $100 \%$ (Fleiss 1971; see Landis and Koch 1977, for a classification of interrater agreement) except for the rarely used dimension acceptance in relation to blood pressure feedback (with $\kappa=.52$ and $99 \%$ agreement). Agreement for the residual category was fair to moderate with $\kappa=.23(90 \%)$ and $\kappa=.52(94 \%)$ for reactions to blood pressure and cholesterol feedback, respectively. Disagreements were resolved by discussion among the raters before further analyses.

\section{Results}

Frequency of spontaneous reactions

In total, participants generated one to four reactions after receiving risk feedback. The average number of reactions to blood pressure feedback was $M=1.35$ ( $\mathrm{SD}=0.57$ ), and a comparable amount of reactions was reported after cholesterol feedback $(M=1.38, \mathrm{SD}=0.63, F(1)=2.36$, ns). A single reaction (e.g., "I am happy"; "It is good") was generated by $68 \%$ and $70 \%$ of the participants after cholesterol and blood pressure feedback, respectively. Multiple reactions (e.g., "The result was lower than I expected", "I think that I am benefiting from controlling my dietary habits and exercising") were generated by $32 \%$ (cholesterol) and 30\% (blood pressure) of the participants. For both risks, the number of reactions generated did not differ significantly between participants receiving a normal test result and those who had an elevated test result ( $p$ s $>.55$ ). The mean number of reactions generated is highly comparable to results reported for Western samples (e.g., $M=1.33$ thoughts reported by Orbell and Hagger 2006; $M=1.26$ thoughts reported by Orbell et al. 2004).

Types of reactions: network nodes

In a first step towards gaining information about the types of spontaneous reactions and to develop a network of reactions to health risk feedback in a real-life setting, each reaction dimension coded in the RFR (e.g., emotions) was translated into a network node (cf. Figs. 1, 2). Thus, each network node represents one of the nine broad types of reactions to individual health risk feedback. In addition, each network node was scaled in accordance to the observed prevalence of the represented reaction type in order to illustrate its practical relevance.

\section{Blood pressure}

As Fig. 1 shows, emotions clearly constituted the most prevalent type of spontaneous reactions, generated by $39 \%$ of the participants. Reactions related to risk feedback valence were generated second most frequently, i.e., by 


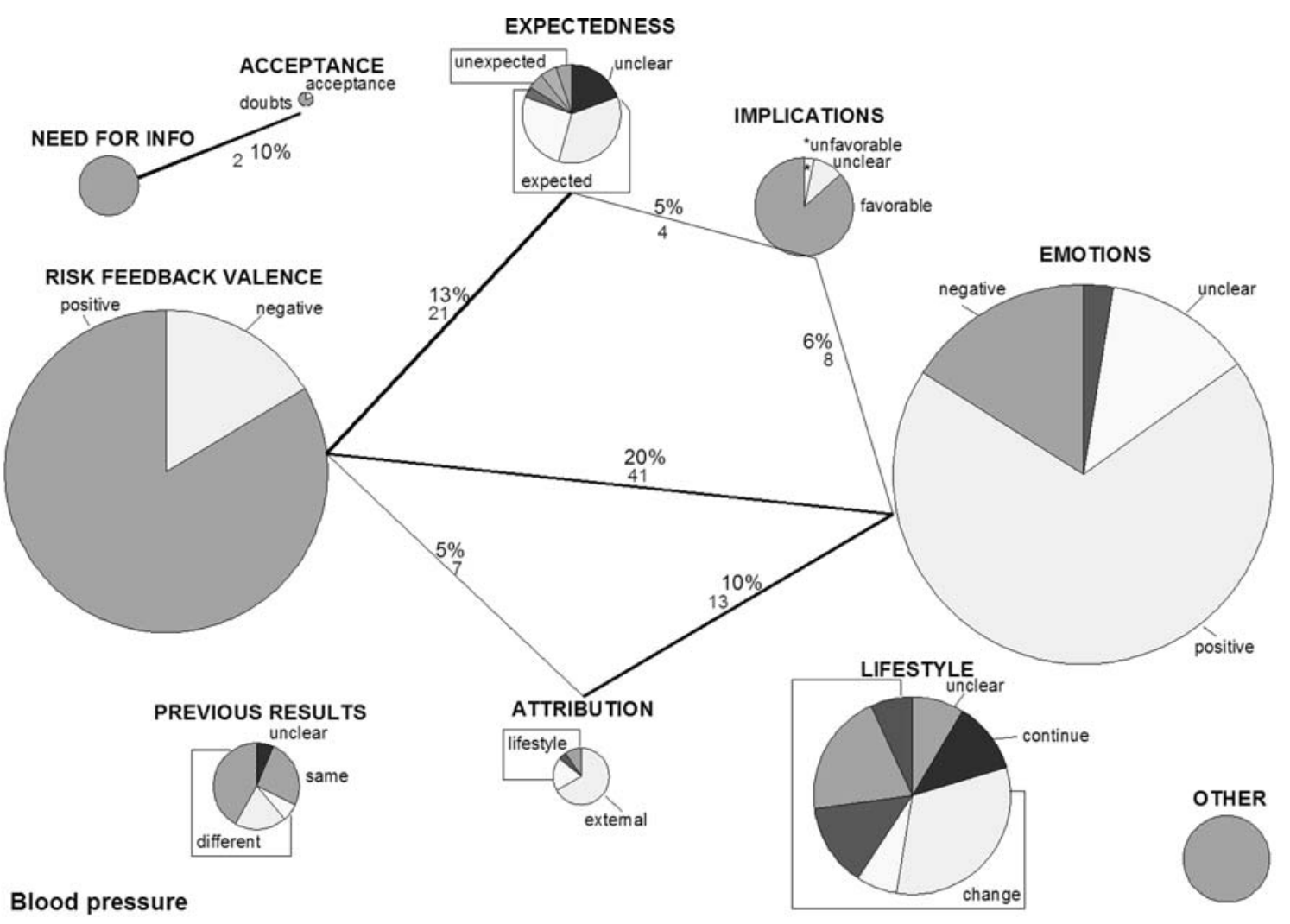

Fig. 1 Network of spontaneous reactions to blood pressure feedback. Multiple responses were possible. Circles (and circle segments) are sized according to the relative frequency with which each reaction category (and subcategory) was generated. Numbers indicate the

$33 \%$. The third most frequent reaction type encompassed reactions pertaining to future lifestyle, generated by $20 \%$ of participants in response to the blood pressure feedback. The reaction types expectedness and implications for health were generated spontaneously by $10 \%$ of the participants. Each other reaction type was generated spontaneously by less than $10 \%$ of participants. Accordingly, the reaction types causal attributions and comparisons with previous results form comparatively small nodes in the reaction network for blood pressure feedback. Even smaller nodes emerged for need for information and acceptance, as these reactions were generated by less than $5 \%$ of participants.

\section{Cholesterol}

Reactions toward cholesterol feedback (cf. Fig. 2) mirrored the pattern found for blood pressure feedback, with emotional reactions $(31 \%)$, feedback valence $(26 \%)$, lifestyle change $(15 \%)$, and expectedness $(15 \%)$ as the most frequent reaction types. Other reaction types such as causal attributions, implications for health, and comparisons with previous results again formed comparatively small nodes in the network (5 8\%). Even smaller nodes emerged for the absolute and relative frequencies of reaction pairs. Percentages were calculated relative to the total of persons who generated either one or both reactions within the respective pair

types need for information and acceptance, as these reactions were generated by less than $5 \%$ of participants after receiving cholesterol feedback. Comparing the absolute frequencies of reactions toward cholesterol and blood pressure feedback showed small, albeit significant differences for risk feedback valence, emotions, and expectedness $\left(F \mathrm{~s}(1)<8.8, p \mathrm{~s}<.04, \eta \mathrm{s}_{\mathrm{p}}^{2}<.02\right)$.

\section{Control analyses}

After both blood pressure and cholesterol feedback, the various reaction types were generated similarly frequently by those who received optimal feedback and those who did not $\left(\mathrm{X}^{2} \mathrm{~s}(1)<2.7, p \mathrm{~s}>.07\right)$. The only exception are reactions pertaining to future lifestyle, which were generated more frequently by those who received non-optimal feedback compared with those who received favorable feedback $\left(\mathrm{X}^{2} \mathrm{~s}(1)>9.1, p \mathrm{~s}<.01\right)$.

Additional control analyses showed that the types of reactions generated were highly similar among those participants who had converging risk feedback across both waves of the study (i.e., optimal feedback at both first and second screening, or repeated non-optimal feedback) and those who had received diverging risk feedback (i.e., optimal 


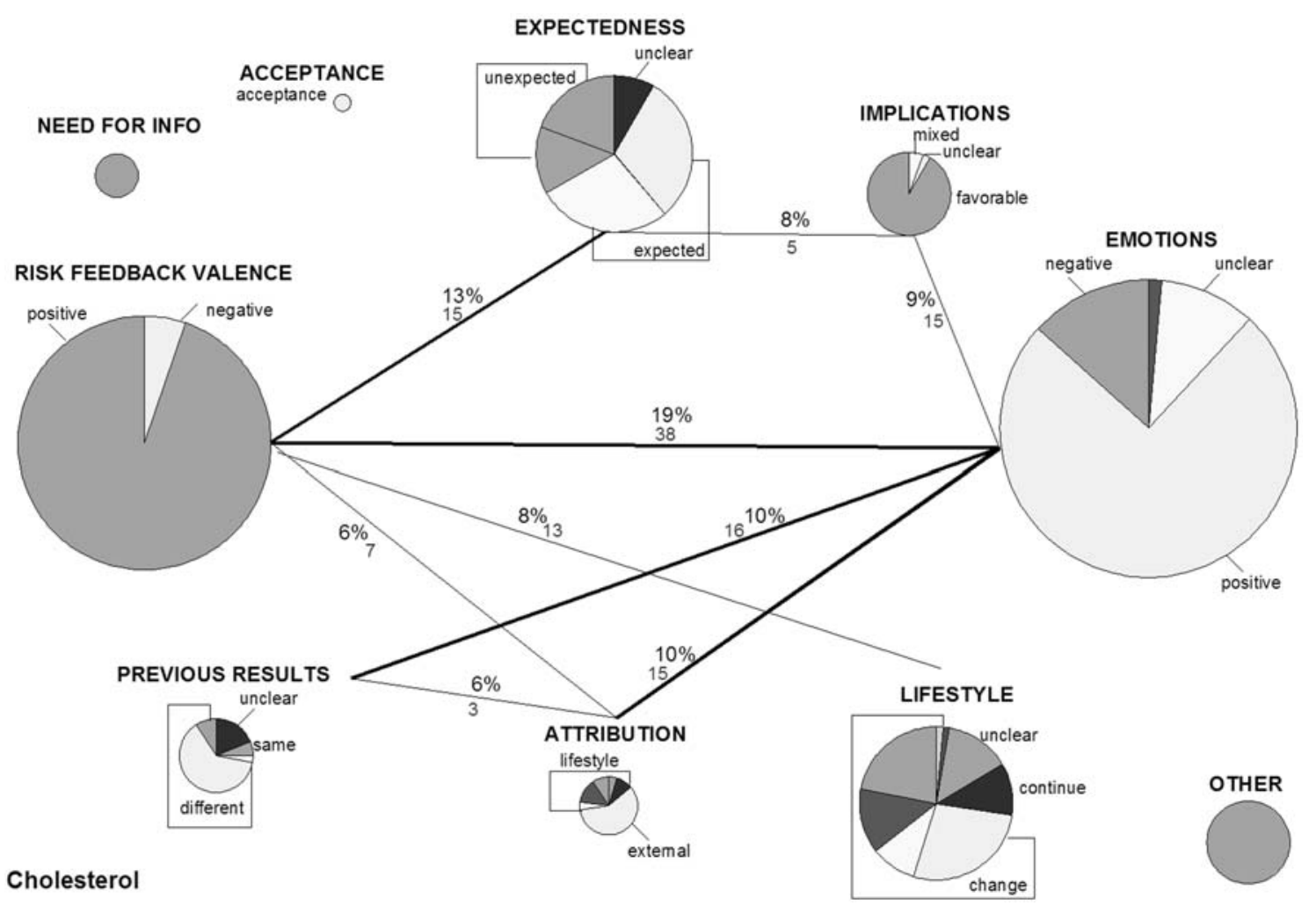

Fig. 2 Network of spontaneous reactions to blood pressure feedback. Multiple responses were possible. Circles (and circle segments) are sized according to the relative frequency with which each reaction category (and subcategory) was generated. Numbers indicate the absolute and relative frequencies of reaction pairs. Percentages were calculated relative to the total of persons who generated either one or both reactions within the respective pair feedback at wave 1 , but non-optimal feedback at wave 2 , or vice versa). The only exception were again reactions concerning future lifestyle, which were generated more frequently by recipients of divergent feedback for both blood pressure and cholesterol feedback $\left(X^{2} \mathrm{~s}(1)>4.34\right.$, $p s<.04)$.

In accordance with Panzer and Renner (2008), younger adults (below 35 years) and older adults (35+ years) generated largely similar reaction types: Reactions constituting large nodes (emotions, risk feedback valence, expectedness, and future lifestyle) and small nodes (implications, need for information, and acceptance) were highly similar across the two age groups. Only the following comparisons were significant: Adults older than 35 years were less likely to generate risk feedback valence or implications for health than younger adults and more likely to generate thoughts about future lifestyle in response to cholesterol or blood pressure feedback $\left(X^{2} \mathrm{~s}(1)>4.64, p \mathrm{~s}<.03\right)$.

Specific content of reaction types: node segmentation

In a next step, each network node was further segmented according to the specific form of reaction demonstrated.
The segments were sized in proportion to the frequency with which the respective reaction occurred (cf. Figs. 1, 2).

\section{Blood pressure}

As Fig. 1 shows, the emotion node consists of four different segments: positive emotions (69\% of the total node), negative emotions (16\%), mixed emotions (2\%), and unclear references to emotions (13\%). The feedback valence node entails a large segment for reactions referring to a positive valence ( $84 \%$ of the node) and a small segment with reactions referring to a negative valence (16\%). Reactions pertaining to the expectedness of the received test result were allotted to seven different subcategories. Three of these seven reaction subcategories referred to expected results (high or low as expected, as expected without reference to the specific value). They jointly account for $65 \%$ of the node. Sixteen percent of the node encompasses reactions referring to unexpected results (higher or lower than expected, different than expected without reference to the direction). The remaining $19 \%$ of the node represent reactions with an ambiguous reference to the expectedness of the result. The node for future 
lifestyle consists of a prominent group of segments for lifestyle change ( $80 \%$ of the node), a smaller segment for lifestyle continuation (12\%), and an even smaller segment for reactions with an ambiguous reference to future lifestyle (8\%). The group of segments for lifestyle change entails responses for the following subcategories: Proposed changes in nutrition ( $7 \%$ of the total node) or exercise (20\%), other specific lifestyle changes such as check-ups (14\%), change of multiple specific lifestyle aspects (7\%, e.g., "I need to drink less and exercise more"), and unspecific lifestyle changes $(32 \%$, e.g., "Let's be more careful"). The node for reactions related to implications for future health consists of a large segment for favorable implications (87\%), and a smaller one for unfavorable implications (3\%). A third residual segment encompasses reactions related to future health without mentioning valence $(10 \%)$. The reaction node for comparisons of current and previous test result includes a large segment representing reactions which point to a perceived difference between current and previous results (68\%, with $42 \%$ stating that the current result is more negative, $19 \%$ for more positive current results, and $7 \%$ for different results without clear indication of their valence). A smaller segment consists of comparisons indicating similarity between former and current results (26\%), and an additional segment contains responses unclearly related to previous test results $(6 \%)$. Within the causal attribution node, two main segments were created, one for external attributions $(67 \%$ of the node), and one consisting of several smaller segments for attributions to lifestyle aspects (33\%, with 5\% for nutrition, $9 \%$ for exercise, and $19 \%$ for attributions to a single other lifestyle aspect).

\section{Cholesterol}

As Fig. 2 shows, highly similar results were found for cholesterol feedback. The largest segment of the emotion node consists of positive emotions ( $75 \%$ of the node). Negative emotions (13\%), mixed emotions (1\%) and unclear references to emotions (11\%) were again seldom mentioned. For the feedback valence node reactions pertaining to a positive valence outnumbered those referring to a negative valence, with ratios of 95:5\%. The node for the expectedness of the test result includes: (1) expected results $58 \%$ of the total node, with $28 \%$ high as expected and $30 \%$ without reference to valence), (2) unexpected results ( $34 \%$ of the node, with $20 \%$ lower than expected and $14 \%$ without reference to valence), and (3) reactions related to expectedness without clear indication of whether the result was expected or not ( $8 \%$ of the node). The node for implications for future health includes a very large segment for positive implications (92\% of the node) whereas no negative implications were mentioned. Mixed implications (5\%), and implications whose valence remained unclear $(3 \%)$ were mentioned only rarely. The node for reactions related to future lifestyle shows that most reactions $(75 \%)$ referred to lifestyle change (with $22 \%$ nutrition change, $14 \%$ exercise change, $1 \%$ changes in smoking, $1 \%$ changes in alcohol consumption, $10 \%$ changes of more than one specific lifestyle aspect, and $27 \%$ unspecific lifestyle change). Comparably few reactions entailed a lifestyle continuation (11\%) or remained unclear in respect to whether change or maintenance was proposed $(14 \%)$. The node for comparisons of current with previous test result again consists of a large segment indicating a difference between current and previous results $(68 \%$ of the node, with $9 \%$ for more negative current results, $56 \%$ for more positive current results, and $3 \%$ for different current results without reference to their valence). A second segment of $6 \%$ pertains to similarity between previous and current results, and a third segment of $19 \%$ contains responses referring to previous results without indicating whether they are similar or different to current results. Within the causal attribution node a larger segment consists of external attributions, which account for $59 \%$ of the total node. A smaller segment includes attributions to lifestyle accounting for $41 \%$ (with $14 \%$ for nutrition, $9 \%$ for exercise, $5 \%$ for a single lifestyle aspect for which no specific subcategory was available, $9 \%$ for multiple lifestyle aspects, and $4 \%$ for unspecific lifestyle attributions).

Interrelations between reaction types: network paths

In a third step, the interrelations between pairs of reaction types were assessed by examining how often two broad reaction types were mentioned jointly within one response to the thought listing task. In Figs. 1, 2, these interrelations are displayed as network paths. In general, two reactions were more likely to be generated jointly if their base rates were high. Therefore, in addition to reporting the absolute number of recipients who jointly generated the two reactions, prevalences adjusted for reaction base rates are reported. Each network path is thus not only superscribed with the base rate of jointly generated reactions, but also with the percentage of joint generations relative to the total of persons who generated at least one of the two reactions connected by the specific path (cf. Figs. 1, 2). Reactions generated jointly by more than $10 \%$ of those who mentioned at least one of the two reactions within each pair are connected by a path drawn as a thick line. Paths with joint generations of less than $10 \%$ but more than $5 \%$ are drawn as a thin line, and paths with joint generations of less than $5 \%$ are omitted for greater clarity.

The results show that emotions and feedback valence were quite often generated conjointly. Among those who generated either emotions or thoughts about feedback 
valence, almost one-fifth generated both reactions. Secondly, of those who generated thoughts pertaining to risk feedback valence or to the expectedness of the received test result, $13 \%$ generated both reactions conjointly. Thirdly, among recipients who generated emotions or causal attributions, $10 \%$ generated both.

Two additional paths were found either only for blood pressure feedback or for cholesterol feedback, but not for both types of risk feedback. For blood pressure feedback, $10 \%$ of those who generated cognitions about need for information or acceptance of the given feedback generated both types of reactions conjointly. For cholesterol feedback, $10 \%$ of those who reported emotions or compared the current and previous cholesterol test result generated both reactions jointly (cf. Figs. 1, 2).

All other reaction pairs were generated by less than $10 \%$ of those who mentioned at least one reaction per pair.

Patterns of reactions toward positive and negative risk feedback

In the last step, the match between reactions and the given feedback (positive vs. negative) was analyzed in order to identify adaptive and maladaptive reaction patterns in relation to the valence of the received feedback. In order to do so, responses containing at least one specific reaction related to emotions, risk feedback valence, implications, and future lifestyle change were analyzed. From a normative point of view, recipients receiving an elevated reading should be more likely to generate negative reactions towards the received feedback including reactions such as negative emotions, and thoughts that negatively evaluate the negative feedback or its implications for their health. Most importantly, recipients of negative feedback should also be inclined to change their lifestyle in the future rather than to continue it. Conversely, recipients of positive feedback should be more likely to express positively toned emotions and they should evaluate the feedback valence and its implications in a positive way. As a consequence, they should be more inclined to continue with their lifestyle.

The remaining responses such as reactions related to expectedness, causal attribution, need for information, and acceptance cannot be categorized as being more or less adaptive in relation to the feedback valence from a normative point of view. For example, both positive and negative feedback could be equally expected or unexpected. Hence, the following analyses are focused on reactions containing at least one clear normative reference point to feedback valence such as emotions, risk feedback valence, implications, and future lifestyle change. In total, 105 responses to blood pressure feedback and 84 responses after cholesterol feedback were thus classifiable as either adaptive or maladaptive.

Participants receiving a normal test result demonstrated in $96 \%$ (blood pressure) and 91\% (cholesterol) of the observed cases an adaptive response pattern: They reported positive emotions or implications for health, or described their feedback as desirable, without generating negative emotions, negative implications, negative risk feedback valence, or intentions to change their lifestyle. A comparably small proportion of $4 \%$ (blood pressure) to $9 \%$ (cholesterol) of those who received a normal test result showed a maladaptive reaction pattern: Despite having received a favorable test result, they generated neither positive emotions, positive implications, nor positive risk feedback valence, but at least one of the specific negative reactions (negative emotions, implications, or risk feedback valence, or future lifestyle change). Thus, participants receiving a normal reading rarely showed a reaction which could be classified as being maladaptive from a normative perspective.

However, a different picture emerged for participants receiving an elevated test result: In particular, reactions were coded as adaptive if they included negative emotions, negative implications, negative feedback valence, or future lifestyle change without containing positive emotions, positive risk feedback valence, or positive implications. In total, 73\% (blood pressure) and 50\% (cholesterol) demonstrated such an adaptive reaction pattern. A maladaptive response pattern, that is a response pattern which includes at least one positive reaction but no negative one nor plans for future lifestyle change was reported by $27 \%$ (blood pressure) and $50 \%$ (cholesterol) of the participants after receiving negative feedback.

\section{Discussion}

The aim of the present study was to examine spontaneous reactions to individual health risk feedback in a real-life setting from a network perspective. For both cholesterol and blood pressure feedback a highly similar reaction pattern emerged, indicating a high reliability of the findings.

Reaction types: how do people react after receiving risk feedback?

Turning to the question of what types of reactions people demonstrate after receiving self-relevant health feedback, the present results show that emotions, thoughts about the valence and expectedness of the given feedback, and about one's future lifestyle constitute the most prevalent reaction types. Causal attributions, implications for health, com- 
parisons with previous results, need for information, and acceptance were comparably seldom generated. Since the pattern of reaction types was not only similar across the two risk feedback types but also similar to the results reported by Panzer and Renner (2008), one might speculate that the four reaction types emotions, feedback valence, expectedness, and future lifestyle change might represent the "big four" of spontaneous reactions toward risk feedback. Interestingly, this pattern of results contradicts the attention these reaction types have received so far in research. Previous experimental studies have largely focused on the degree to which recipients accept risk feedback and on the implications they perceive for their future health (cf. Ditto et al. 1998; Ditto and Croyle 1995; Ditto and Lopez 1992). Emotional responses have only recently received attention mainly in the form of negatively valenced reactions such as worry (e.g., De Hoog et al. 2005; French et al. 2006; Lipkus et al. 2004) while positive emotional responses and the expectedness of the given feedback have largely been neglected.

Specific content of reaction types: node segmentation and interrelations

Findings regarding the interrelations between spontaneous reactions showed that emotions, risk feedback valence, and expectedness form large and well-connected network nodes. Interestingly, reactions pertaining to lifestyle change formed an also large, but less well-connected node. Thus, contrary to previous research assuming that certain cognitive reactions such as acceptance of feedback are the pathway to awareness and behavior change, participants seem to chose a more direct, intuitive pathway to behavior change. In current risk research frameworks, intuitive or experiential modes of reactions are discussed (cf. Damasio 2004; Loewenstein et al. 2001; Slovic et al. 2004; Weber and Hsee 1999). Accordingly, people might rely not only on analytic deliberation for decisions but also on affective experiences when assessing the need for behavior change.

At large, the present network can be seen as a first step towards a cognitive affective domain map of reactions to health risk feedback as proposed by the cognitive affective system theory of personality (CAPS, Mischel and Shoda 1995; Shoda and Mischel 2006). Cognitive affective domain maps allow for tracing "personal signatures" of individuals, describing situation transient reactions. Thus, an individual network analysis could extend the typical grouplevel data analysis. A comparison of an individual network with a group-based "social normative" network could be used by researchers, practitioners, and recipients in order to detect potentially critical reaction patterns, such as those indicating maladaptive reactions. For example, recipients with maladaptive patterns after negative feedback might be less likely to adopt behavior change than those with adaptive patterns. Similarly, maladaptive reactions after positive feedback might be precursory to retesting behavior.

\section{Adaptive reaction patterns}

After positive feedback, adaptive patterns were more prevalent than after negative feedback. Thus, recipients of negative feedback tend to express feedback-inconsistent thoughts such as mismatching (i.e., positive) emotions more often than recipients of positive feedback, indicating a maladaptive pattern with reactions which are nonnormative from a medical point of view. This difference in reactions toward positive and negative feedback is explained from a Motivated Reasoning perspective (Kunda 1990) as indicating self-defensive reactions which help to maintain a positive view of one's own health. However, even after negative feedback, adaptive reaction patterns were far more prevalent than maladaptive ones. This suggests that despite a possible self-defensive pattern on the group-level, most recipients of negative feedback do properly acknowledge the valence of their result, as for example reflected by their spontaneous report of negative emotions. Generally, reactions to health risk feedback seem to be predominately adaptive, supporting previous research (e.g., Renner 2004). The patterns found in the present study are, however, drawn from initial reactions towards health risk feedback, and their adaptivity was only assessed in relation to the valence of the received feedback. However, in order to assess the long-term adaptivity of the found reaction patterns, long-term outcomes including actual health behavior need to be assessed (cf. Renner et al. 2008; Sweeny and Shepperd 2007).

\section{Limitations and suggestions for future research}

The present study was limited to the exploration of spontaneous reactions to feedback about two risk factors for CVD. Results were highly similar for both risk factors indicating a satisfactory reliability of our findings. However, future research is needed to examine whether the pattern of results can be generalized to other health risks. The present network is based on open-ended verbal responses, thereby providing a measure for initial spontaneous reactions that are consciously accessible to the participants. However, the array of reactions towards feedback certainly also includes implicit reactions which are by definition not accessible to self-reports. A promising avenue for future research might be to combine different methods such as self-report and physiological measures in order to capture explicit and implicit reaction patterns. The network was derived from reactions to a second wave health risk feedback. While the 
generated reaction types were largely similar to those after the first feedback (cf. Panzer and Renner 2008), we cannot rule out that the adaptivity of reactions is impacted by the familiarity with the feedback. Thus, future research is needed to explore the potential unfolding of reactions across repeated risk feedback.

There are cultural differences between our South Korean sample and previously researched Western samples (e.g., Croyle et al. 2006; Ditto et al. 2003, cf. also Ditto and Croyle 1995), which might have partly contributed to a low degree of self-defensiveness in our sample (cf. Heine and Hamamura 2007; but see also Sedikides et al. 2007). While there is some evidence that Westerners might also process self-relevant health risk feedback in an adaptive way (Renner 2004; cf. also De Hoog et al. 2005; Liberman and Chaiken 2003), empirical studies indicate that Eastern samples may be in general less prone to self-biased information processing. Thus, the present South Korean sample might not be representative in terms of the amount of adaptive reactions of European or North American samples. However, multiple group comparisons of reactions to health risk feedback between representative samples from different cultures need to be conducted in order to draw more exhaustive conclusions on the adaptivity of reactions across cultures.

The present results were moreover obtained within a relatively healthy sample. In a less healthy sample, we would probably have found different base rates on the level of specific reactions, e.g., more negative emotions and less positive ones. We would, however, expect similar results with regard to the broad reaction types generated, their base rates (cf. Panzer and Renner 2008), and their interrelations. Due to the relatively small number of recipients of non-optimal feedback, our findings regarding the proportion of adaptive reaction patterns within this group need to be interpreted cautiously.

The present study used a network approach to explore spontaneous reactions to health risk feedback for the first time. While we believe that this approach has yielded interesting results from a real-life setting, one of the most promising future direction could be the application of a network approach in an experimental setting, e.g., using the TAA paradigm (Croyle and Ditto 1990). This would allow for a more rigorous design, randomization of positive and negative feedback, and overall increased internal validity, thereby providing valuable information about the health risk reaction network.

\section{Implications}

The network of reactions to health risk information could be an effective tool in guiding both research and practice. From a research-oriented perspective, the network could facilitate the selection of core reactions for future studies at the individual and group levels. It can also be used for examining inter-individual differences in the reception of health risk feedback. From a practiceoriented perspective, it could support practitioners in pinpointing reactions which are relevant to their clients. The network could also be used to identify clients with maladaptive reaction patterns, who could then be offered different interventions than those with adaptive reaction patterns might receive. Thus, the network could facilitate the choice of interventions appropriate for the individual patient's needs.

Acknowledgments This research was supported by a Deutsche Forschungsgemeinschaft grant for Britta Renner and Ralf Schwarzer (Grant Re 1583/2 1). The study was part of the RACK Project. Other participating investigators from the RACK study group include: Sunkyo Kwon, Hallym University; Byung Hwan Yang, Hanyang University; Ki Chung Paik, Dankook University. We would like to thank Freda Marie Hartung, Marija Jaroslavskaja, Andries Oeberst, Youlia Spivak, and Dimitar Stoilov for their help in categorizing the responses and James Moon for translating the responses.

\section{References}

Asia Pacific Cohort Studies Collaboration. (2002). Blood pressure indices and cardiovascular disease in the Asia pacific region: A pooled analysis. Hypertension, 42, 6975 .

Asia Pacific Cohort Studies Collaboration. (2005). A comparison of lipid variables as predictors of cardiovascular disease in the Asia pacific region. Annals of Epidemiology, 15, 405 413. doi:10.1016/ j.annepidem.2005.01.005.

Cacioppo, J. R., \& Petty, R. E. (1981). Social psychological procedures for cognitive response assessment: The thought listing technique. In T. Merluzzi, C. Glass, \& M. Genest (Eds.), Cognitive assessment (pp. 309 342). New York: Guilford Press.

Colman, A. M. (2006). A dictionary of psychology. Oxford, NY: Oxford University Press.

Croyle, R. T., \& Ditto, P. H. (1990). Illness cognition and behavior: An experimental approach. Journal of Behavioral Medicine, 13(1), 31 52. doi:10.1007/BF00844898.

Croyle, R. T., Loftus, E. F., Barger, S. D., Sun, Y. C., Hart, M., \& Gettig, J. (2006). How well do people recall risk factor test results? Accuracy and bias among cholesterol screening partic ipants. Health Psychology, 25, 425 432. doi:10.1037/0278 6133. 25.3.425.

Croyle, R. T., Sun, Y. C., \& Louie, D. H. (1993). Psychological minimization of cholesterol test results: Moderators of appraisal in college students and community residents. Health Psychology, 12, 503 507. doi:10.1037/0278 6133.12.6.503.

Damasio, A. R. (2004). Descartes' error: Emotion, reason, and the human brain. New York: Avon.

De Hoog, N., Stroebe, W., \& De Wit, J. B. (2005). The impact of fear appeals on processing and acceptance of action recommenda tions. Personality and Social Psychology Bulletin, 31, 2433. doi:10.1177/0146167204271321.

Ditto, P. H., \& Croyle, R. T. (1995). Understanding the impact of risk factor test results: Insights from a basic research program. In R. T. Croyle (Ed.), Psychosocial effects of screening for disease prevention and detection (pp. 144 181). London: Oxford University Press. 
Ditto, P. H., Jemmott, J. B., \& Darley, J. M. (1988). Appraising the threat of illness: A mental representational approach. Health Psychology, 7, 183 201. doi:10.1037/0278 6133.7.2.183.

Ditto, P. H., \& Lopez, D. F. (1992). Motivated skepticism: Use of differential decision criteria for preferred and nonpreferred conclusions. Journal of Personality and Social Psychology, 63, 568 584. doi:10.1037/0022 3514.63.4.568.

Ditto, P. H., Munro, G. D., Apanovitch, A. M., Scepansky, J. A., \& Lockhart, L. K. (2003). Spontaneous skepticism: The interplay of motivation and expectation in responses to favorable and unfavorable medical diagnoses. Personality and Social Psychol ogy Bulletin, 29, 1120 1132. doi:10.1177/0146167203254536.

Ditto, P. H., Scepansky, J. A., Munro, G. D., Apanovitch, A. M., \& Lockhart, L. K. (1998). Motivated sensitivity to preference inconsistent information. Journal of Personality and Social Psychology, 75, 53 69. doi:10.1037/0022 3514.75.1.53.

Fleiss, J. L. (1971). Measuring nominal scale agreement among many raters. Psychological Bulletin, 76, 378 382. doi:10.1037/h0031619.

French, D. P., Maissi, E., \& Marteau, T. M. (2006). The psychological costs of inadequate cervical smear test results: Three month follow up. Psycho Oncology, 15, 498 508. doi:10.1002/pon.980.

Heckerman, D. (2001). Bayesian graphical models and networks. In N. J. Smelser \& P. B. Baltes (Eds.), International encyclopedia of the social \& behavioral sciences (pp. 1048 1052). Oxford: Pergamon.

Heine, S. J., \& Hamamura, T. (2007). In search of East Asian self enhancement. Personality and Social Psychology Review, 11, 1 24. doi:10.1177/1088868306294587.

Kang, E., Choi, E. J., Song, H. J., Ryou, K. C., Kim, N. Y., Kim, K. N., et al. (2006). Korea national health and nutrition examina tion survey (KNHANES III) 2005 Health behaviors of adults. Seoul, Republic of Korea: Korea Institute of Health and Social Affairs \& Ministry of Health and Welfare.

Kunda, Z. (1990). The case for motivated reasoning. Psychological Bulletin, 108, 480 498. doi:10.1037/0033 2909.108.3.480.

Landis, R. J., \& Koch, G. G. (1977). The measurement of observer agreement for categorical data. Biometrics, 33, 159 174. doi: 10.2307/2529310.

Leventhal, H., Brissette, I., \& Leventhal, E. A. (2003). The common sense model of self regulation of health and illness. In L. D. Cameron \& H. Leventhal (Eds.), The self regulation of health and illness behaviour (pp. 43 65). New York: Routledge.

Liberman, A., \& Chaiken, S. (2003). Defensive processing of personally relevant health messages. In P. Salovey \& A. J. Rothman (Eds.), Social psychology of health (pp. 118 129). New York: Psychology Press.

Lipkus, I. M., McBride, C. M., Pollak, K. I., Lyna, P., \& Bepler, G. (2004). Interpretation of genetic risk feedback among African American smokers with low socioeconomic status. Health Psy chology, 23, 178 188. doi:10.1037/0278 6133.23.2.178.

Loewenstein, G. F., Weber, E. U., Hsee, C. K., \& Welch, N. (2001). Risk as feelings. Psychological Bulletin, 127, 267 286. doi: 10.1037/0033 2909.127.2.267.

Mischel, W., \& Shoda, Y. (1995). A cognitive affective system theory of personality: Reconceptualizing situations, dispositions, dynamics, and invariance in personality structure. Psychological Review, 102, 246 268. doi:10.1037/0033 295X.102.2.246.

Orbell, S., \& Hagger, M. (2006). Temporal framing and the decision to take part in type 2 diabetes screening: Effects of individual differences in consideration of future consequences on persua sion. Health Psychology, 25(4), 537548.
Orbell, S., Perugini, M., \& Rakow, T. (2004). Individual differences in sensitivity to health communications: Consideration of future consequences. Health Psychology, 23(4), 388396.

Organization for Economic Co Operation and Development. (2005). Thematic review on adult learning: Korea country note. Paris: Organization for Economic Co Operation and Development.

Panzer, M., \& Renner, B. (2008). To be or not to be at risk: Spontaneous reactions to risk information. Psychology \& Health, 23, 617627. doi:10.1080/08870440701606889.

Pear, R. (2008, January 18). Growth of genetic tests concerns federal panel. The New York Times. Retrieved 19 June 2008, from http://www.nytimes.com/2008/01/18/us/18tests.html.

Renner, B. (2003). Hindsight bias after receiving self relevant health risk information: A motivational perspective. Memory (Hove, England), 11, 455 472. doi:10.1080/09658210244000531.

Renner, B. (2004). Biased reasoning: Adaptive responses to health risk feedback. Personality and Social Psychology Bulletin, 30, 384 396. doi:10.1177/0146167203261296.

Renner, B., Schüz, B., \& Sniehotta, F. (2008). Preventive health behaviour and adaptive accuracy of risk perceptions. Risk Analysis, 28, 741 748. doi:10.1111/j.1539 6924.2008.01047.x.

Renner, B., Spivak, Y, Kwon, S., \& Schwarzer, R. (2007). Age and health behaviour change: Differences in predicting physical activity of South Korean adults. Psychology and Aging, 22, 482 493. doi: 10.1037/0882 7974.22.3.482.

Sedikides, C., Gaertner, L., \& Vevea, J. L. (2007). Inclusion of theory relevant moderators yield the same conclusions as Sedikides, Gaertner, and Vevea (2005): A meta analytic reply to Heine, Kitayama, and Hamamura (2007). Asian Journal of Social Psychology, 10, 59 67. doi:10.1111/j.1467 839X.2007. 00212.x.

Shoda, Y., \& Mischel, W. (2006). Applying meta theory to achieve generalisability and precision in personality science. Applied Psychology: An International Review, 55, 439 452. doi:10.1111/ j.1464 0597.2006.00264.x.

Slovic, P., Finucane, M. L., Peters, E., \& MacGregor, D. G. (2004). Risk as analysis and risk as feelings: Some thoughts about affect, reason, risk, and rationality. Risk Analysis, 24, 311 322. doi: 10.1111/j.0272 4332.2004.00433.x.

Sudman, S., Bradburn, N. M., \& Schwarz, N. (1996). Thinking about answers: The application of cognitive processes to survey methodology. San Francisco: Jossey Bass.

Sweeny, K., \& Shepperd, J. A. (2007). Being the best bearer of bad tidings. Review of General Psychology, 11, 235 257. doi:10.1037/ 1089 2680.11.3.235.

Taylor, S. E. (1991). Asymmetrical effects of positive and negative events: The mobilization minimization hypothesis. Psychologi cal Bulletin, 110, 67 85. doi:10.1037/0033 2909.110.1.67.

Weber, E. U., \& Hsee, C. K. (1999). Models and mosaics: Investigating cross cultural differences in risk perception and risk preference. Psychonomic Bulletin \& Review, 6, 611617.

Weiner, B. (1985). "Spontaneous" causal thinking. Psychological Bulletin, 97, 74 84. doi:10.1037/0033 2909.97.1.74.

World Health Organization. (2002). The world health report 2002: Reducing risks, promoting healthy life. Geneva, Switzerland: World Health Organization.

World Health Organization. (2004). The atlas of heart disease and stroke. Geneva, Switzerland: World Health Organization. 